\title{
Anaerobic Bacteria in Subjects with Chronic Periodontitis and In Periodontal Health
}

\author{
AK Mane, AP Karmarkar, RS Bharadwaj
}

\section{ABSTRACT}

The aim of the present study was to look at the frequency of strict anaerobic bacteria in patients with chronic periodontitis and healthy subjects without periodontal destruction. Hundred patients with chronic periodontitis and hundred healthy subjects with no clinical signs of periodontal disease were included in the study. Sterile paper points were used for sample collection from the deepest pocket site in case of periodontitis patients. The samples were cultured under anaerobic conditions and characterized to species level by conventional biochemical tests. Anaerobes were isolated in 83\% of periodontitis cases and 62\% of healthy subjects. Porphyromonas gingivalis (48\%), Fusobacterium nucleatum (24\%), Peptostreptococcus micros (23\%) and Prevotella spp. (26\%) were the commonest anaerobes isolated in periodontitis patients, while Peptostreptococcus micros (36\%), Prevotella spp. (8\%), Veillonella (10\%) and Actinomyces viscosus (12\%) were frequently detected in the control group. Data shows the diversity of anaerobic bacteria in chronic periodontitis.

Key-words: Chronic periodontitis, Anaerobic bacteria, Periodontal health

\section{Contact Author}

\section{Dr. Arati Mane}

E-mail : arati2478mane@rediffmail.com
$\mathrm{P}$ eriodontitis is a disease that affects the tooth supporting tissues (periodontium) and is characterized by loss of periodontal attachment including the alveolar bone.(1) At least forty-eight specific periodontitis categories are now recognized. By far the most common is chronic periodontitis and this is the major cause of tooth loss in the adult population.(2) It is generally chronic in nature and can persist in the absence of treatment.(3) The etiology of the disease is multifactorial and bacterial deposits play an essential role in the pathogenesis. The bacteria that are involved in periodontitis accumulate in the sub-gingival plaque that comprises predominantly of Gram-negative strict anaerobic rods.(1) Among them Porphyromonas gingivalis, Prevotella intermedia, Fusobacterium nucleatum, Bacteroides spp., Selenomonas spp. have been associated with chronic or refractory periodontitis. However, some anaerobic, Gram positive microorganisms such as Peptostreptococcus micros and Eubacterium species have recently been implicated in chronic periodontitis.(2) Therefore microbiological laboratory procedures are involved in diagnosis and therapy control of periodontitis. Microbiological findings are important prognostic markers predicting whether attachment gain will be stable or not.(4) Many of these organisms may also be present in periodontally healthy individuals and can exist in communal harmony with the host.(5) Most information about periodontal microbiota has emerged from studies carried out in Europe and USA. Only recently have the periodontal microflora been examined in some third world countries and the results indicate differences both qualitatively and quantitatively when compared to those perceived in the west.(6) Data on anaerobic periodontal microflora in the Indian population is very scarce.

The purpose of this study was to look at the frequency of strict anaerobic bacteria in patients with chronic periodontitis and healthy subjects without periodontal destruction.

\section{Methods}

The patients for the following study were selected from the outpatient Department of Dentistry, B.J.Medical College, Pune, Maharashtra. 


\section{Study Population}

The study population included hundred consecutive patients with chronic periodontitis. All patients showed clinical and radiological evidence of alveolar loss and periodontal pockets depth equal to or exceeding $5 \mathrm{~mm}$. Hundred healthy subjects without clinical signs of periodontal disease were also selected. None of the periodontal patients or healthy subjects had received antibiotics for three months prior to sample collection. Both groups were age and gender matched.

Informed and written consent of all subjects was taken for the study. The study was approved by the Ethics Committee of our institute.

\section{Collection of samples \\ Periodontitis subjects}

Samples were collected with complete aseptic precautions with the assistance of dentists. Initially the site of sample collection was isolated with cotton rolls, carefully cleaned with sterile cotton pellets, and air-dried. For single sites, two sterile paper points were inserted to the bottom of the pocket for a 20 -s period and then transferred to Robertson's cooked meat medium. For pooled samples, at least one paper point per site from upto four sites was collected.(5)

\section{Healthy subjects}

The sub-gingival material collected from healthy subjects with sterile paper points and then transferred to Robertson's cooked meat medium.(6)

\section{Processing of samples}

The samples were processed within 24 hours. For isolation of strict anaerobes, the samples were plated on non-selective blood agar plates (5\%) supplemented with hemin and menadione. Kanamycin-Vancomycin blood agar plates were used for selective recovery of obligate anaerobic Gram-negative rods. The plates were incubated in Lapiz anaerobic jar at $37^{\circ} \mathrm{C}$ under anaerobic conditions for 7 days. After 7 days of incubation, colonies with differing characteristics were subjected to various tests. Identification was based on cell morphology, Gram stain reaction, biochemical and enzymatic tests including catalase, oxidase, indole hydrolysis, esculin hydrolysis, gelatin hydrolysis, urea hydrolysis and fermentation of sugars. All strict anaerobes were identified according to flow charts by Baron and Citron $\operatorname{MD}(8)$ and Koneman et al.(9)

\section{Statistical analysis}

Chi-square test or the Fischer's Exact test were used for determining the differences in frequencies of various periodontal pathogens in chronic periodontitis patients and healthy subjects. $\mathrm{p}$ value of $<0.05$ was considered as statistically significant.

\section{Results}

Anaerobes were isolated in $83 \%$ of periodontitis cases and $62 \%$ of healthy subjects. Polymicrobial infection was seen in $72.28 \%$ cases. 2 isolates were found in $91.66 \%$ while 3 in $8.2 \%$ cases. Table 1. shows the spectrum of anaerobes in patients with periodontitis and control group subjects and the statistical analysis.

\section{Discussion}

The results of present study show the diversity of anaerobic bacteria in chronic periodontitis patients. Anaerobic bacteria were isolated in $83 \%$ of periodontitis cases and $62 \%$ of the healthy subjects. Various studies from India and other countries showed an isolation rate of strict anaerobes ranging from 42 to $100 \%$ in periodontitis cases and 38 to $80 \%$ in healthy subjects. $(1,5,7)$ The varying recovery rates of isolation can be due to varying criteria of patient selection, culture method employed, geographical differences and the molecular techniques used for identification.(7) Polymicrobial pattern of infection is routinely encountered in periodontitis. In the present study polymicrobes were found in $72.28 \%$ cases of

\begin{tabular}{|c|c|c|c|}
\hline Organisms isolated & Periodontitis patientsN (\%) & Healthy subjectsN (\%) & pvalue \\
\hline \multicolumn{4}{|l|}{ Gram negative organisms } \\
\hline Porphyromonas gingivalis & $48(48 \%)$ & $12(12 \%)$ & $<0.01$ \\
\hline Prevotella melanogenica & $14(14 \%)$ & $06(6 \%)$ & 0.016 \\
\hline Prevotella intermedia & $12(12 \%)$ & $02(2 \%)$ & 0.028 \\
\hline Fusobacterium nucleatum & $24(24 \%)$ & $08(8 \%)$ & $<0.05$ \\
\hline Bacteroides spp. & $05(5 \%)$ & 00 & N.S. \\
\hline Veillonella spp. & $09(9 \%)$ & $10(10 \%)$ & N.S. \\
\hline \multicolumn{4}{|l|}{ Gram positive organisms } \\
\hline Peptostreptococcus micros & $23(23 \%)$ & $36(36 \%)$ & N.S. \\
\hline Peptostreptococcus productus & $04(4 \%)$ & 00 & N.S. \\
\hline Streptococcus intermedius & $05(5 \%)$ & 00 & N.S. \\
\hline Actinomyces viscosus & $02(2 \%)$ & $12(12 \%)$ & N.S. \\
\hline Eubacterium lentum & $02(2 \%)$ & $04(4 \%)$ & N.S. \\
\hline
\end{tabular}


which 2 isolates were found in $91.66 \%$ while 3 isolates in $8.2 \%$ cases. Salari and Saini et al have brought out the polymicrobial nature of periodontal flora in their studies. $(5,7)$

In the present study Gram negative anaerobes $(75.7 \%)$ were predominantly isolated than the Gram positive (44.1\%) in the periodontitis cases. Porphyromonas gingivalis (48\%), Fusobacterium nucleatum (24\%), Peptostreptococcus micros $(23 \%)$ and Prevotella spp. (26\%) were the commonest organisms isolated. Other workers have isolated similar anaerobes but in varying proportions.(1,5,7) Van Winkelhoff et al has reported a much higher number of these isolates [Porphyromonas gingivalis (59.5\%), Fusobacterium nucleatum (95.7\%), Peptostreptococcus micros (94\%) and Prevotella intermedia (87.9\%)] while Salari et al has reported relatively lower number of these isolates [Porphyromonas gingivalis (21.9\%), Fusobacterium nucleatum (0.4\%), Peptostreptococcus micros $(1.3 \%)$ and Prevotella intermedia (10.5\%)].(1,5) This variations can be attributed to geographical differences. Porphyromonas gingivalis (12\%),Peptostreptococcus micros (36\%), Prevotella spp. (8\%), Veillonella (10\%) and Actinomyces viscosus (12\%) were frequently detected in the control group. In the study carried out by Saini et al in India has reported similar isolates except Porphyromonas gingivalis or Prevotella spp.(7)

The mere presence of pathogen alone is not sufficient to cause disease but they need to be present above a determined threshold to cause periodontal disease. This associated with low levels of beneficial species, environmental and genetic factors leads to increased susceptibility of periodontitis.(10) In our study Porphyromonas gingivalis, Prevotella melanogenica, Prevotella intermedia and Fusobacterium nucleatum were isolated more frequently in periodontitis patients than the control group and the results were statistically significant $(p<0.05)$. Thus these species are the strongest markers of periodontal destruction in our study. Van Winkelhoff et al in his study reported Porphyromonas gingivalis, Prevotella intermedia, Bacteroides forsythus, Fusobacterium nucleatum and Prevotella melaninogenica to be significantly more prevalent in adult periodontitis cases than in controls.(1)

\section{Conclusion}

Data of the present study shows the diversity of anaerobic bacteria in chronic periodontitis which should be considered in the treatment strategy of the patients. Porphyromonas gingivalis, Prevotella melanogenica, Prevotella intermedia and Fusobacterium nucleatum were the strongest markers of periodontal destruction in our study. Cultural methods for recovery of bacteria are still gold standard in microbiology. However many bacteria in the oral cavity cannot be cultured, it is likely that these still uncharacterized bacteria might play a role in the initiation and progression of periodontal disease. Considering the scarce data on microbial flora in the Indian population further studies for assessment of microbial profile in various forms of periodontitis should be carried out.

\section{Acknowledgement}

We would like to thank the entire staff of the Department of Dentistry for their support rendered throughout the study.

\section{THE AUTHORS}

\section{Mane AK}

M.D.

Lecturer

Dept. of Microbiology, B.J. Medical College, Pune, Maharshtra, India

\section{Correspondence Author}

9, Vijay Housing Society, University Road,

Pune-411016, Maharashtra, India

Ph: 09422349348, E-mail: arati2478mane@ rediffmail.com

\section{Karmarkar AP}

M.D.

Associate Professor

Dept. of Microbiology, B.J. Medical College, Pune, Maharshtra, India

\section{Bharadwaj RS}

M.D.

Professor and Head of Department

Dept. of Microbiology, B.J. Medical College, Pune, Maharshtra, India

\section{References}

1. VanWinkelhoff AJ, Loss BG, Van der Reijden WA, Van der Velden $U$. Porphyromonas gingivalis, Bacteroides forsythus and other periodontal pathogens in subjects with and without periodontal destruction. J Clin Periodontol 2002;29:1023-1028.

2. C, Nonnenmacher R Mutters, L. Flores de Jacoby. Microbiological characteristics of subgingival microbiota in adult periodontitis, localized juvenile periodontitis and rapidly progressive periodontitis subjects. Clin Microbiol Infect 2001;7:213-217.

3. Daniluk T, Tokajuk G, Cylwik-Rokicka D, Rozkiewicz D, Zaremba ML, Stokowska W. Aerobic and anaerobic bacteria in subgingival and supragingival plaques of adult patients with periodontal disease. Advances in Medical Sciences 2006;51(1):81-85.

4. Eick S, Pfister W. Comparison of microbial cultivation and a commercial nucleic acid based method for detection of peroiodontopathogenic species in subgingival plaque samples. $J$ Clin Periodontol 2002;29:638-644.

5. Mohammad Hossein Salari, Zainab Kadkhoda. Rate of cultivable subgingival periodontopathogenic bacteria in chronic periodontitis. Journal of Oral Science 2004;46(3):157-161.

6. Joshi VM, Vandana KL. The detection of eight putative periodontal pathogens in adult and rapidly progressive periodontitis patients. Indian J Dent Res 2007;18:6-10.

7. Saini Aparna, N Gupta, A Mahajan, DR Arora. Microbial flora in orodental infections. Indian $J$ of Medical Microbiology 2003;21(2):111-114.

8. Baron EJ, Citron DM. Anaerobic infection flow charts using minimal laboratory resources. Clin Infec Dis 1997;25(2):143-46.

9. Koneman EW, Allen SD, Janda MW, Schreckenberger PC. The anaerobic bacteria. Color Atlas and Textbook Of Diagnostic Microbiology, $5^{\text {th }}$ edition, 1997 ,Philadelphia, Lippincott JB, Ch 14, 725-727.

10. Colombo, Ana Paula V. Subgingival microbiota of Brazilian subjects with untreated chronic periodontitis. J Periodontol 2002;73:360369. 